\title{
Effects of reaction progress variable definition on the Flame Surface Density transport statistics and closure for different combustion regimes
}

\author{
Vassilios Papapostolou ${ }^{1}$, Nilanjan Chakraborty ${ }^{1}$, Markus Klein ${ }^{2 *}$, Hong G. Im $^{3}$ \\ ${ }^{1}$ School of Engineering \\ University of Newcastle \\ Claremont Road, Newcastle \\ NE1 7RU, UK \\ Email: V.S.Papapostolu1@ncl.ac.uk ; nilanjan.chakraborty@ncl.ac.uk \\ ${ }^{2}$ Universität der Bundeswehr München, Fakultät für Luft- und Raumfahrttechnik, LRT1, \\ Werner-Heisenberg-Weg 39, 85577 Neubiberg, Germany \\ Email: markus.klein@unibw.de \\ ${ }^{3}$ Clean Combustion Research Center, \\ King Abdullah University of Science and Technology (KAUST), \\ Thuwal 23955-6900, Saudi Arabia \\ Email: hong.im@kaust.edu.sa
}

* Corresponding author 


\section{ABSTRACT}

The implications of the choice of reaction progress variable on the performances of the Flame Surface Density (FSD) based mean reaction rate closure and the well-established sub-models of the FSD transport have been analysed in context of Reynolds Averaged Navier Stokes simulations. For this purpose, a detailed chemistry Direct Numerical Simulation (DNS) database of freely-propagating $\mathrm{H}_{2}$-air flames (with an equivalence ratio of 0.7 ) spanning the corrugated flamelets, thin reaction zones and broken reaction zones regimes of premixed turbulent combustion has been considered. The FSD and the unclosed terms of its transport equation have been analysed for reaction progress variables defined based on normalised $\mathrm{H}_{2}, \mathrm{O}_{2}$ and $\mathrm{H}_{2} \mathrm{O}$ mass fractions and temperature. The performances of the closures for turbulent flux of FSD, and tangential strain rate term have been found to be mostly unaffected by the choice of reaction progress variable. However, the well-established existing models for the unresolved tangential strain rate term have been found not to perform well for the cases representing the corrugated flamelets and thin reaction zones regimes of premixed combustion. The performance of a well-established existing model for the combined propagation and curvature terms has been found to be significantly dependent on the choice of reaction progress variable. Furthermore, the surface-averaged value of the density-weighted displacement speed cannot be approximated by the corresponding unstretched laminar flame value especially for the flames in the broken reaction zones regime. Detailed explanations have been provided for the observed behaviours of the FSD based reaction rate closure and sub-models for the unclosed terms of the FSD transport equation in different combustion regimes for different choices of reaction progress variable.

Keywords: Flame Surface Density, Reynolds Averaged Navier Stokes simulations, Reaction progress variable, Reaction rate closure, Direct Numerical Simulations 


\section{INTRODUCTION}

The mean reaction rate closure in premixed turbulent combustion is often achieved using the flame surface density (FSD), defined as the flame surface area per unit volume (Candel and Poinsot, 1990). The generalised FSD is defined as (Boger et al., 1998): $\Sigma_{g e n}=\overline{|\nabla c|}$ where $c$ is the reaction progress variable (RPV) and the overbar indicates a Reynolds averaging or filtering operation in the context of Reynolds-averaged Navier-Stokes (RANS) or large eddy simulations (LES). A number of previous analyses focussed on both algebraic (Boger et al., 1998; Cant and Bray, 1998; Charlette et al., 2002; Knikker et al., 2002; Keppeler et al., 2014; Klein et al., 2016; Chakraborty and Klein., 2008a; Ma et al., 2013) and transport equation (Cant et al., 1990; Candel et al., 1990; Duclos et al., 1993; Veynante et al., 1996; Hawkes and Cant 2001a; Chakraborty and Cant, 2007; Hun and Huh, 2008; Katragadda et al., 2011; Chakraborty et al., 2011; Hernandez-Perez et al., 2011; Reddy and Abraham, 2012; Chakraborty and Cant, 2013; Ma et al., 2014; Sellman et al., 2017) based closures of FSD for turbulent premixed combustion. However, most of these analyses (Boger et al., 1998; Cant and Bray, 1998; Charlette et al., 2002; Keppeler et al., 2014; Klein et al., 2016; Chakraborty and Klein., 2008a; Ma et al., 2013; Cant et al., 1990; Candel et al., 1990; Duclos et al., 1993; Hawkes and Cant 2001a; Chakraborty and Cant, 2007; Hun and Huh, 2008; Katragadda et al., 2011; Chakraborty et al., 2011; Hernandez-Perez et al., 2011; Reddy and Abraham, 2012; Chakraborty and Cant, 2013; Ma et al., 2014; Sellman et al., 2017) have been carried out based on single-step Arrhenius type chemistry and the effects of detailed chemical mechanism on the statistical behaviour of FSD and its transport are yet to be analysed in detail.

With the advances in computing power, it turned out that the well-known modelling challenges arising from the differential diffusion of heat and mass may be addressed by solving individual scalar transport equations with detailed chemistry models in RANS (Colin et al., 2003) or even 
in LES (Li and Kong 2008, Vermorel et al., 2009). Moreover, Colin et al. (2003) and Vermorel et al. (2009) solved transport equations for several major species, whereas the mean/filtered reaction rate has been closed by transporting one single FSD which may not behave necessarily similarly for all species. Thus, it is necessary to analyse the differences in the FSD transport for various definitions of the reaction progress variable (RPV) in a multi-species system.

Since FSD models were originally based on simple chemistry and in the corrugated flamelet (CF) regime, it is important to assess (i) their validity with detailed chemistry and (ii) their validity beyond the CF regime. As for (i), one of the most crucial aspects is the representation of the flame structures using different definitions of the reaction progress variable (RPV) associated with different species variables, and the present study is one of the first attempts to address this issue in detail. As for (ii), while the original FSD formulation was developed in the CF regime, in principle the generalised FSD $\Sigma_{g e n}=\overline{|\nabla c|}$ is a field variable (Boger et al., 1998) and thus should be applicable irrespective of the regime. This raises the fundamental question about the possibility of using the original model under non-flamelet conditions, such as in the broken reaction zones regime, prior to any modifications are proposed.

For a multi-species system, various definitions of the RPV, $c$, are possible depending on the choice of the scalar variable. The influences of the choice of $c$ on the statistical behaviour of $\Sigma_{g e n}$ and its modelling are yet to be discussed in open literature. The present analysis addresses the aforementioned gaps in the existing literature by assessing the FSD based reaction rate closure and the well-established sub-models of the FSD transport in the context of RANS using a three-dimensional direct numerical simulation (DNS) database of $\mathrm{H}_{2}$-air flames with an equivalence ratio of 0.7 (which ensures that the flames remain globally thermo-diffusively neutral, in that the flame speed is insensitive to stretch (Im and Chen, 2002)). The simulation 
parameters for this DNS database have been chosen such that the cases considered here represent typical combustion situations within the corrugated flamelets $(\mathrm{CF})$, thin reaction zones (TRZ) and broken reaction zones (BRZ) regimes of premixed turbulent combustion. The main objectives of the present analysis are:

(1) to analyse the statistical behaviour of the FSD transport in different regimes of premixed turbulent combustion,

(2) to assess the performances of well-established existing closures for the FSD transport for different definitions of $c$.

The mathematical background and numerical implementation pertaining to this analysis are detailed in the next section. The results will be presented in the following section and subsequently discussed. The main findings are summarised and conclusions are drawn in the final section of this paper.

\section{MATHEMATICAL BACKGROUND \& NUMERICAL IMPLEMENTATION}

The RPV is defined as: $c=\left(Y_{0}-Y\right) /\left(Y_{0}-Y_{\infty}\right)$, where $Y$ is the mass fraction of a chosen species, and subscripts 0 and $\infty$ indicate the values in the unburned and fully burned gases, respectively. The RPV can alternatively be defined based on temperature as $c=$ $\left(T-T_{0}\right) /\left(T_{a d}-T_{0}\right)$ where $T, T_{0}$ and $T_{a d}$ are the instantaneous, unburned gas and adiabatic flame temperatures respectively. All definitions of $c$ are identical if the Lewis number is unity for all species in the case of a single-step chemical mechanism; however, they differ in real flames due to the differential diffusion effects. In this analysis, RPVs based on temperature, $\mathrm{H}_{2}, \mathrm{O}_{2}$ and $\mathrm{H}_{2} \mathrm{O}$ mass fractions are considered for $\mathrm{H}_{2}$-air flames with an equivalence ratio of 0.7. This selection is not based on the fact that these species would represent the best definition of RPV for hydrogen or hydrocarbon flames. However, all of these species can be used to 
define valid RPVs and they represent a bandwidth of Lewis numbers ranging from considerably smaller $\left(\mathrm{H}_{2}\right)$ to considerably larger than unity $\left(\mathrm{O}_{2}\right)$. The transport equation of $\tilde{c}$ is given by:

$$
\partial(\bar{\rho} \tilde{c}) / \partial t+\partial\left(\bar{\rho} \tilde{u}_{j} \tilde{c}\right) / \partial x_{j}=\overline{\dot{w}+\partial\left[\rho D\left(\partial c / \partial x_{\jmath}\right)\right] / \partial x_{\jmath}}-\partial\left(\overline{\rho u_{\jmath}^{\prime \prime} c^{\prime \prime}}\right) / \partial x_{j}
$$

where $\rho$ is the gas density, $u_{j}$ is the $\mathrm{j}^{\text {th }}$ component of velocity, $\tilde{q}=\overline{\rho q} / \bar{\rho}$ and $q^{\prime \prime}=q-\tilde{q}$ are the Favre average and fluctuation of a general variable $q$, respectively. Here, $\dot{w}=$ $-\dot{w}_{Y} /\left(Y_{0}-Y_{\infty}\right)\left(\dot{w}=\dot{w}_{T} / C_{P}\left(T_{a d}-T_{0}\right)\right)$ for mass fraction (temperature) based RPV with $\dot{w}_{Y}$ $\left(\dot{w}_{T}\right)$ being the net reaction rate of the corresponding species (the heat release rate), and $C_{P}$ is the specific heat at constant pressure.

The terms on the right hand side of eq. 1 are unclosed. The first two terms on the RHS can be modelled as (Boger et al., 1998): $\overline{\dot{w}+\nabla \cdot(\rho D \nabla c)}=\overline{\left(\rho S_{d}\right)_{s}} \Sigma_{g e n}$ where $\left.\overline{(q)}\right)_{s}=\overline{q|\nabla c|} / \Sigma_{g e n}$ is the surface-averaged value of a general quantity $q$ and $S_{d}=|\nabla c|^{-1}(D c / D t)=[\dot{w}+$ $\nabla \cdot(\rho D \nabla c)] / \rho|\nabla c|$ is the displacement speed. In the context of RANS, $\bar{w} \gg$ $\overline{\partial\left[\rho D\left(\partial c / \partial x_{J}\right)\right] / \partial x_{J}}$ (Chakraborty et al., 2011) and thus $\overline{\dot{w}}$ can be closed if $\Sigma_{g e n}$ and $\overline{\left(\rho S_{d}\right)_{s}}$ are appropriately evaluated. In order to solve eq. 1, the turbulent scalar flux components $\left(\overline{\rho u_{\jmath}{ }^{\prime \prime} c^{\prime \prime}}\right)$ need to be modelled but this aspect is mostly independent of the FSD transport and its closure, and thus the discussion in this paper does not explicitly deal with the modelling of turbulent flux components. The closures of $\overline{\rho u_{\jmath}{ }^{\prime \prime} c^{\prime \prime}}$ have been discussed elsewhere in detail (Veynante et al., 1997; Chakraborty and Cant, 2009a,b) and thus will not be discussed in this paper. The closures of sub-grid scalar flux and Reynolds scalar flux for the database considered here can be found in Klein et al. (2018) and Papapostolou et al. (2018), respectively, and thus are not repeated here. 
The transport equation for $\Sigma_{g e n}$ takes the following form (Cant et al., 1990; Candel et al., 1990; Duclos et al., 1993; Veynante et al., 1996; Hawkes and Cant 2001a; Chakraborty and Cant, 2007; Hun and Huh, 2008; Katragadda et al., 2011; Chakraborty et al., 2011; Hernandez-Perez et al., 2011; Reddy and Abraham, 2012; Chakraborty and Cant, 2013; Ma et al., 2014; Sellman et al., 2017):

$$
\begin{aligned}
& \partial \Sigma_{g e n} / \partial t+\partial\left(\tilde{u}_{j} \Sigma_{g e n}\right) / \partial x_{j}=\underbrace{-\partial\left\{\left[\overline{\left(u_{k}\right)_{s}}-\tilde{u}_{k}\right] \Sigma_{\text {gen }}\right\} / \partial x_{k}}_{T_{1}-\text { turbulent transport }}+ \\
& \underbrace{\overline{\left(\left(\delta_{l \jmath}-N_{l} N_{\jmath}\right) \partial u_{l} / \partial x_{J}\right)_{s}} \Sigma_{g e n}}_{T_{2} \text {-strain rate }} \underbrace{-\partial\left[\overline{\left(S_{d} N_{k}\right)_{s}} \Sigma_{\text {gen }}\right] / \partial x_{k}}_{T_{3}-\text { propagation }}+\underbrace{\overline{\left(S_{d} \partial N_{l} / \partial x_{l}\right)_{s}} \Sigma_{\text {gen }}}_{T_{4}-\text { curvature }}
\end{aligned}
$$

where $N=-\nabla c /|\nabla c|$ is the local flame normal vector. The distributions of $\nabla c$ and $S_{d}$ are different for each scalar used for the RPV definition, and this is considered while evaluating all the terms of eq. 2 .

A three-dimensional DNS (Arias et al., 2016; Wacks et al., 2016) database of $\mathrm{H}_{2}$-air flames with an equivalence ratio of 0.7 , employing a detailed chemical mechanism (Burke et al., 2012) with 9 species and 19 chemical reactions, is considered here. The unburned gas temperature $T_{0}$ is taken to be $300 \mathrm{~K}$, which leads to an unstrained laminar burning velocity $S_{L}=135.6 \mathrm{~cm} / \mathrm{s}$ and heat release parameter $\tau=\left(T_{a d}-T_{0}\right) / T_{0}=5.71$ (where $T_{a d}$ is the adiabatic flame temperature) under atmospheric pressure. The numerical implementation pertaining to this database has been discussed elsewhere (Arias et al., 2016; Wacks et al., 2016) in detail and thus will not be repeated here. Turbulent inflow and outflow boundaries are taken in the direction of mean flame propagation and transverse boundaries are taken to be periodic. The inflow and outflow boundaries are specified using an improved version of the Navier Stokes characteristic boundary conditions (NSCBC) technique (Yoo et al., 2005). The inflow turbulent velocity fluctuations are specified by scanning a plane through a frozen turbulent homogeneous 
isotropic incompressible velocity fluctuation field, which was generated using a pseudospectral method (Rogallo, 1981) following the Passot-Pouquet spectrum (Passot and Pouquet, 1987). The temporal evolution of flame area has been monitored and the flame is considered to be statistically stationary when the flame area no longer varies with time. The inflow values of normalised root-mean-square turbulent velocity fluctuation $u^{\prime} / S_{L}$, turbulent length scale to flame thickness ratio $l_{T} / \delta_{t h}$, Damköhler number $D a=l_{T} S_{L} / u^{\prime} \delta_{t h}$, Karlovitz number $K a=$ $\left(\rho_{0} S_{L} \delta_{t h} / \mu_{0}\right)^{0.5}\left(u^{\prime} / S_{L}\right)^{1.5}\left(l_{T} / \delta_{t h}\right)^{-0.5}$ and turbulent Reynolds number $R e_{t}=\rho_{0} u^{\prime} l_{T} / \mu_{0}$ for all cases are presented in Table 1 where $\mu_{0}$ is the unburned gas viscosity, $\delta_{t h}=\left(T_{a d}-\right.$ $\left.T_{0}\right) / \max |\nabla T|_{L}$ is the thermal flame thickness and the subscript ' $\mathrm{L}$ ' is used to refer to unstrained laminar flame quantities. Cases A-C are representative of the $\mathrm{CF}(K a<1)$, TRZ $(1<K a<$ $100)$ and BRZ $(K a>100)$ regimes (Peters, 2000) of premixed combustion respectively.

The domain size is $20 \mathrm{~mm} \times 10 \mathrm{~mm} \times 10 \mathrm{~mm}(8 \mathrm{~mm} \times 2 \mathrm{~mm} \times 2 \mathrm{~mm})$ in cases A and B (case C) and the domain has been discretised by a uniform Cartesian grid of $512 \times 256 \times 256$ $(1280 \times 320 \times 320)$. Simulations have been carried out for $1.0 t_{e}, 6.8 t_{e}$ and $6.7 t_{e}$ (i.e. $t_{e}=$ $l_{T} / u^{\prime}$ ) for cases A-C respectively, and this simulation time remains comparable to several previous analyses (Boger et al., 1998; Charlette et al., 2002; Hun and Huh, 2008; Reddy and Abraham, 2012).

\section{RESULTS \& DISCUSSION}

The distributions of RPV for the flames considered here are presented elsewhere (Arias et al., 2016; Wacks et al., 2016) and thus are not shown here. In statistically planar flames, $\tilde{c}$ is a unique function of the mean direction of flame propagation and thus all the terms are presented here as functions of $\tilde{c}$ for different definitions of RPV. 


\section{Statistical behaviour of the unclosed terms of the FSD transport equation}

The variations of normalised values of $T_{1}-T_{4}$ with $\tilde{c}$ for cases A-C are shown in Fig. 1 for different choices of RPV. Figure 1 shows that $T_{2}$ and $T_{4}$ predominantly act as the dominant source and sink terms respectively for all cases irrespective of the choice of RPV. However, $T_{4}$ assumes positive values for $\tilde{c}<0.2$ in case $\mathrm{A}$, whereas this term remains negative throughout the flame brush for cases $\mathrm{B}$ and $\mathrm{C}$ for all choices of RPV. The contributions of $T_{1}$ and $T_{3}$ assume both positive and negative values within the flame brush. The relative magnitudes of $T_{1}$ and $T_{3}$ in comparison to those of $T_{2}$ and $T_{4}$ remain small in the cases representing the TRZ and $\mathrm{BRZ}$ regimes (i.e. cases $\mathrm{B}$ and $\mathrm{C}$ ), whereas the magnitudes of $T_{1}, T_{2}$ and $T_{3}$ remain comparable in the case representing the $\mathrm{CF}$ regime (i.e. case A). The above observation holds for all the different choices of RPV considered here. In cases B and C, the magnitudes of positive $T_{2}$ and negative $T_{4}$ remain comparable, whereas the magnitude of $T_{2}$ remains small in comparison to $T_{4}$ in case A for all definitions of RPV considered here. The dominant behaviour of $T_{2}$ and $T_{4}$ in all cases are consistent with scaling estimates presented earlier by Chakraborty and Cant (Chakraborty and Cant, 2013) in a simple chemistry DNS analysis. The implications of the choice of RPV on the modelling of $T_{1}-T_{4}$ will be discussed next in this section.

\section{Modelling of the turbulent transport term $T_{1}$}

The modelling of $T_{1}$ depends on the closure of the turbulent flux of FSD $\left[\left(u_{l}\right)_{s}-\tilde{u}_{l}\right] \Sigma_{g e n}$. A gradient transport (GT) hypothesis $\left[\overline{\left(u_{k}\right)_{s}}-\widetilde{u_{k}}\right] \Sigma_{g e n}=-\left(v_{t} / S_{c_{\Sigma}}\right) \partial \Sigma_{g e n} / \partial x_{k}\left(\right.$ where $v_{t}=$ $C_{\mu} \tilde{k}^{2} / \tilde{\varepsilon}$ is the eddy kinematic viscosity and $S_{c_{\Sigma}}$ is the turbulent Schmidt number with $\tilde{k}=$ $\overline{\rho u_{\imath}^{\prime \prime} u_{\imath}^{\prime \prime}} / 2 \bar{\rho}$ and $\tilde{\varepsilon}=\overline{\mu\left(\partial u_{\imath}^{\prime \prime} / \partial x_{\jmath}\right)\left(\partial u_{\imath}^{\prime \prime} / \partial x_{\jmath}\right)} / \bar{\rho}$ being the turbulent kinetic energy and its dissipation rate, and $C_{\mu}=0.09$ is a model parameter) is often employed for the closure of turbulent flux of FSD (Hawkes and Cant 2001a; Hernandez-Perez et al., 2011; Ma et al., 2014). 
However, previous analyses (Chakraborty et al., 2011; Chakraborty and Cant, 2013; Sellman et al., 2017) suggested that the turbulent flux of FSD exhibits a counter-gradient behaviour when the turbulent flux of RPV $\overline{\rho u_{1}^{\prime \prime} c^{\prime \prime}}$ shows a counter-gradient transport (CGT) and vice versa. Chakraborty and Cant (2011) modified an existing model (Veynante et al., 1997) in order to make it capable of predicting both GT and CGT of FSD as:

$$
\left[\overline{\left(u_{l}\right)_{S}}-\tilde{u}_{l}\right] \Sigma_{g e n}=(1-2 \tilde{c}) \overline{\rho u_{l}^{\prime \prime} c^{\prime \prime}} \Sigma_{g e n} /\left[\overline{\rho c^{\prime \prime 2}}+\bar{\rho} \tilde{c}(1-\tilde{c})\right]
$$

The predictions of eq. 3 are shown alongside $\left[{\overline{\left(u_{l}\right)_{s}}}_{-}-\tilde{u}_{l}\right] \Sigma_{g e n}$ extracted from DNS data in Fig. 2 for cases A-C for all the different choices of RPV. Figure 2 indicates that the GT model does not adequately capture both the qualitative and quantitative behaviours of $\left[\overline{\left(u_{l}\right)_{S}}-\widetilde{u_{l}}\right] \Sigma_{g e n}$ in cases A and B for all choices of RPV. Furthermore, in these cases the GT model predicts the wrong sign of $\left[\overline{\left(u_{l}\right)_{S}}-\widetilde{u}_{l}\right] \Sigma_{g e n}$ in some locations of the flame brush, which is indicative of a CGT of $\Sigma_{\text {gen }}$. In contrast, eq. 3 is more successful in capturing the qualitative behaviour of $\left[{\overline{\left(u_{l}\right)_{s}}}_{-}-\tilde{u}_{l}\right] \Sigma_{g e n}$ despite having quantitative differences between the model prediction and DNS data for all cases irrespective of the choice of the RPV. A reasonable level of agreement between the predictions of the GT model and DNS data is obtained for case C for all choices of RPV and the performance of the GT model in this case remains comparable to that of the model given by eq. 3. The qualitative agreement between the GT model prediction and $\left[\overline{\left(u_{l}\right)_{s}}-\tilde{u_{l}}\right] \Sigma_{g e n}$ in case $\mathrm{C}$ suggests a GT of $\Sigma_{g e n}$ in this case. A CGT is obtained when flame normal acceleration dominates over turbulent fluctuations which can be quantified as $\tau S_{L}>u^{\prime}$ (Veynante et al., 1997) where $\tau=\left(T_{a d}-T_{0}\right) / T_{0}$ is the heat release parameter which is 5.71 for this database. Accordingly, cases A and B (where $\tau S_{L}>u^{\prime}$ ) exhibit a CGT of $\Sigma_{g e n}$, whereas a GT is obtained for case $\mathrm{C}$ (where $\tau S_{L}<u^{\prime}$ ). The observations from Fig. 2 suggest that eq. 3 is capable of predicting predominantly CGT (GT) for cases A and B (case C). 


\section{Modelling of the tangential strain rate term $T_{2}$}

The term $T_{2}$ is often modelled by splitting it into two components (Cant et al., 1990; Candel et al., 1990; Duclos et al., 1993; Veynante et al., 1996; Hawkes and Cant 2001a):

$$
T_{2}=\underbrace{\left[\delta_{i k}-\overline{\left(N_{l} N_{k}\right)_{s}}\right]\left(\partial \tilde{u}_{l} / \partial x_{k}\right) \Sigma_{g e n}}_{T_{21}}+\underbrace{\overline{\left(\left[\delta_{l k}-N_{l} N_{k}\right] \partial u_{l}^{\prime \prime} / \partial x_{k}\right)_{s}} \Sigma_{g e n}}_{T_{22}}
$$

Cant et al. (1990) proposed $\quad{\overline{\left(N_{l} N_{k}\right)_{s}}}={\overline{\left(N_{l}\right)_{s}}}_{\overline{\left(N_{k}\right)_{s}}}+\left(\delta_{i k} / 3\right)\left[1-{\overline{\left(N_{l}\right)}}_{s} \overline{\left(N_{l}\right)_{s}}\right]$ (CantPope-Bray (CPB) model) where $\overline{\left(N_{k}\right)_{s}}=-\left(\partial \bar{c} / \partial x_{k}\right) / \Sigma_{g e n}$. Chakraborty and Cant (2011) suggested $\bar{c}=\left(1+\tau g^{1.5} L e^{-0.26}\right) \tilde{c} /\left[1+\tau g^{1.5} L e^{-0.26} \tilde{c}\right]\left(\right.$ where $g=\overline{\rho c^{\prime \prime 2}} / \bar{\rho} \tilde{c}(1-\tilde{c})$ is the segregation factor, $L e$ is the Lewis number of the RPV), which is valid for both $D a<1$ and $D a>1$ flamelet combustion, and this expression can be used to evaluate $\overline{\left(N_{k}\right)_{s}}=$ $-\left(\partial \bar{c} / \partial x_{k}\right) / \Sigma_{g e n}$ for all cases for all definitions of RPV (not shown here).

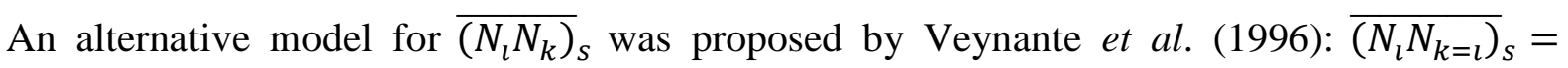
$\sum_{l \neq i} \widetilde{u_{l}^{\prime \prime} u_{l}^{\prime \prime}} / 4 \tilde{k}$ and $\overline{\left(N_{l} N_{k \neq l}\right)_{s}}=\widetilde{u_{l}^{\prime \prime} u_{k}^{\prime \prime}} / 2 \tilde{k}$ (VPDM Model). Figure 3 reveals that the CPB model consistently captures the qualitative behaviour of $T_{21}$ for all choices of RPV, but this model underestimates the magnitude of $T_{21}$. The VPDM model is more successful in terms of capturing the magnitude of $T_{21}$ for all three cases despite a slight overprediction in case $\mathrm{B}$. Experimental (Veynante et al., 1996) and DNS (Chakraborty and Cant, 2006) results suggested that the unresolved part of $\overline{\left(N_{l} N_{k}\right)_{S}}$ does not remain isotropic, as assumed in the CPB model, whereas the VPDM model accounts for the anisotropy of $\overline{\left(N_{l} N_{k}\right)}-{\overline{\left(N_{l}\right)}}_{S}{\overline{\left(N_{k}\right)}}_{s}$. This difference is reflected in the superior performance of the VPDM model in Fig. 3. A similar observation was made by Chakraborty and Cant (2006) in the context of LES modelling using simple chemistry DNS data. 
The two most popular models for $T_{22}$ are the ones proposed by Cant et al. (1990) (i.e. $T_{22}=$ $0.28 \sqrt{\tilde{\varepsilon} / v_{0}} \Sigma_{\text {gen }}$ henceforth referred to as the CPB model) and by Candel et al. (1990) (i.e. $T_{22}=a_{0} \Gamma_{k}(\tilde{\varepsilon} / \tilde{k}) \Sigma_{g e n}$ henceforth referred to as the coherent flamelet model (CFM) model (Duclos et al., 1993) where $v_{0}$ is the kinematic viscosity in the unburned gas and is defined as $v_{0}=\mu_{0} / \rho_{0}$ with $\mu_{0}$ and $\rho_{0}$ being the unburned gas viscosity and density respectively, $a_{0}$ is a model constant (taken as $a_{0}=2.0$ following (Chakraborty et al., 2011; Chakraborty and Cant, 2013)), and $\Gamma_{k}$ is the efficiency function (Meneveau and Poinsot, 1991), which depends on $l_{t} S_{L} / \alpha_{T 0}$ and $\sqrt{2 \tilde{k} / 3} / S_{L}$ with $\alpha_{T 0}$ being the thermal diffusivity in the unburned gas, and $l_{t}=$ $C_{k} \tilde{k}^{3 / 2} / \tilde{\varepsilon}$ is the local integral length scale, where $C_{k}$ depends on the local Taylor micro-scale Reynolds number (Lindstedt and Vaos, 1999).

The predictions of the CPB and CFM models are compared to $T_{22}$ extracted from the DNS data in Fig. 4 for cases A-C for different choices of RPV. Figure 4 shows that both CPB and CFM models significantly overpredict $T_{22}$ for cases $\mathrm{A}$ and $\mathrm{B}$. Moreover, the term $T_{22}$ remains negative in the middle of the flame brush in case $\mathrm{A}$, whereas $T_{22}$ assumes negative values towards the burned gas side of the flame brush before vanishing altogether in case B. In contrast, both CPB and CFM models predict only positive values throughout the flame brush. In case $\mathrm{C}$, the term $T_{22}$ exhibits positive values throughout the flame brush and the qualitative agreement between the DNS data and the model predictions are improved, with the CFM model performing better quantitatively than CPB which overpredicts $T_{22}$ throughout the flame brush.

The term $T_{22}$ can be expressed as: $T_{22}=\overline{\left(e_{\alpha} \sin ^{2} \alpha+e_{\beta} \sin ^{2} \beta+e_{\gamma} \sin ^{2} \gamma\right)|\nabla c|}$ where $e_{\alpha}, e_{\beta}$ and $e_{\gamma}$ are the most extensive, intermediate and the most compressive eigenvalues of $e_{i j}=$ $0.5\left(\partial u_{i}^{\prime \prime} / \partial x_{j}+\partial u_{j}^{\prime \prime} / \partial x_{i}\right)$ and $\alpha, \beta$ and $\gamma$ are the angles between $\nabla c$ and the eigenvectors 
associated with $e_{\alpha}, e_{\beta}$ and $e_{\gamma}$ respectively. It has been demonstrated elsewhere (Chakraborty and Swaminathan, 2007) that $\nabla c$ preferentially aligns collinearly with the eigenvector associated with $e_{\alpha}$ (i.e. high probability of $\sin ^{2} \alpha \approx 0$ ) for $D a \gg 1$ flames (e.g. case A) where the strain rate due to flame normal acceleration overcomes turbulent straining. Thus, $T_{22}=$ $\overline{\left(e_{\alpha} \sin ^{2} \alpha+e_{\beta} \sin ^{2} \beta+e_{\gamma} \sin ^{2} \gamma\right)|\nabla c|}$ is likely to exhibit negative values in cases $\mathrm{A}$ and $\mathrm{B}$. The effects of flame normal straining weaken with increasing $\mathrm{Ka}$ (Chakraborty and Swaminathan, 2007) (e.g. from case A to case B) and thus the likelihood of obtaining negative values of $T_{22}$ is relatively smaller in case $\mathrm{B}$ than in case A. In contrast, $\nabla c$ preferentially aligns collinearly with the eigenvector associated with $e_{\gamma}$ (i.e. high probability of $\sin ^{2} \gamma \approx 0$ ) for $D a<1$ combustion (e.g. case C) where turbulent straining overcomes the strain rate due to flame normal acceleration, and thus, $T_{22}$ exhibits only positive values in case $\mathrm{C}$.

Both CPB and CFM models neglect the effects of strain rate associated with flame normal acceleration (Katragadda et al., 2011). The CPB model scales the strain rate in terms of the Kolmogorov time scale $\left(\sqrt{v_{0} / \tilde{\varepsilon}}\right)$ which is strictly valid for passive material surfaces (Cant $e t$ al., 1990). As flames resemble increasingly passive surfaces at higher $K a$, the CPB model is poor in capturing the behaviour of $T_{22}$ for cases $\mathrm{A}$ and $\mathrm{B}$ but improves for case $\mathrm{C}$. As for the CFM model, which is based on the large-scale turbulent strain rate $\left(\tilde{\varepsilon} / \tilde{k} \sim u^{\prime} / l_{t}\right)$, the change of $\nabla c$ alignment with local principal strain rates induced by flame normal acceleration is not properly accounted for, although it may be argued that $\Gamma_{k}$ can include such information. Moreover, both CPB and CFM models implicitly are based on the preferential collinear alignment between $\nabla c$ with the eigenvector associated with $e_{\gamma}$, which is valid only for case $\mathrm{C}$.

It is worth noting that different efficiency factor $\Gamma_{k}$ expressions have also been proposed in the context of LES (Charlette et al., 2002; Colin et al., 2000) but applying them in the CFM model 
does not improve its prediction significantly in cases A and B, and also does not capture the negative values of $T_{22}$. Chakraborty and Cant (Chakraborty et al., 2011; Chakraborty and Cant, 2013) proposed a correction factor of the form $a_{c o r r}=a_{T 22}(1-\tilde{c})^{\xi} / L e^{p}$ where $a_{T 22}, \xi$ and $p$ are the model parameters for both CPB and CFM models but this is not considered in this analysis because this correction factor is not capable of predicting negative values of $T_{22}$.

It is worth noting that both CPB and CFM models have been proposed and assessed for simple chemistry mechanisms and unity Lewis number (Cant et al., 1990; Candel et al., 1990; Duclos et al., 1993; Hawkes and Cant 2001a; Chakraborty and Cant, 2007; Hernandez-Perez et al., 2011; Reddy and Abraham, 2012; Chakraborty and Cant, 2013; Ma et al., 2014). Moreover, the assumptions behind the CPB and CFM models are also not consistent with the flame physics in cases A-C. For example, the surface-averaged tangential strain rate for the CPB model is taken to scale with the Kolmogorov time scale $\tau_{\eta}=\sqrt{v_{0} / \tilde{\varepsilon}}$, which is valid for material surfaces (Yeung et al., 1990) but not valid for a flame which propagates normal to itself (Cant et al., 1990). By contrast, the CFM model scales with the surface-averaged tangential strain rate with large-scale turbulent straining $\tilde{k} / \tilde{\varepsilon}$ but the efficiency function $\Gamma_{k}$ allows for different extents of flame wrinkling (Meneveau and Poinsot, 1991). However, $\Gamma_{k}$ is calibrated based on two-dimensional unsteady flame-vortex interaction, which is not representative of actual flame-turbulence interaction. Both $\mathrm{CPB}$ and CFM models do not explicitly account for chemical time-scale and $\nabla c$ alignment with local principal strain rates. Although most previous assessments of the CPB and CFM models have been carried out for simple single-step chemistry, the analysis by Chakraborty and Cant (2011, 2013), Katragadda et al. (2011) and Sellmann et al. (2017) also reported unsatisfactory predictions of the CPB and CFM models. Katragadda et al. (2011) and Sellmann et al. (2017) recently modelled $T_{D}=\overline{\left(\partial u_{\imath}^{\prime \prime} / \partial x_{l}\right)}{ }_{s} \Sigma_{g e n}$ and $\left(-T_{N}\right)=-\overline{\left(N_{l} N_{\jmath} \partial u_{l}^{\prime \prime} / \partial x_{\jmath}\right)} \Sigma_{S} \Sigma_{g e n}$ separately, and explicitly accounted for the alignment 
statistics of $\nabla c$ with local principal strain rates, but this approach is not yet established and is beyond the scope of current work.

\section{Modelling of the combined propagation and curvature terms $\left(T_{3}+T_{4}\right)$}

The combined contribution of $\left(T_{3}+T_{4}\right)$ is usually modelled together in the following manner due to their displacement speed $S_{d}$ dependence (Hun and Huh, 2008; Chakraborty et al., 2011; Chakraborty and Cant, 2013; Sellman et al., 2017):

$$
\left(T_{3}+T_{4}\right)=-\frac{\partial}{\partial x_{i}}\left[\frac{\rho_{0} S_{L}}{\bar{\rho}} \overline{\left(N_{l}\right)} s_{s} \Sigma_{g e n}\right]+\frac{\rho_{0} S_{L}}{\bar{\rho}} \frac{\partial \overline{\left(N_{l}\right)} s_{S}}{\partial x_{i}} \Sigma_{g e n}-\beta_{0} \alpha_{N} \frac{\left(\bar{c}-c_{c p}\right) S_{L} \Sigma_{g e n}^{2}}{\bar{c}(1-\bar{c})}
$$

where $\alpha_{N}=1-{\overline{\left(N_{k}\right)}}_{S}{\overline{\left(N_{k}\right)}}_{s}$ and $\beta_{0}$ and $c_{c p}$ are the model parameters (Chakraborty et al., 2011; Chakraborty and Cant, 2013; Sellman et al., 2017). The model parameter $\beta_{0}$ has to satisfy $\beta_{0}>1$ in order to ensure realisability (i.e. $\Sigma_{g e n} \geq 0$ ) (Duclos et al., 1993; Hawkes and Cant, 2001b), and this has been confirmed in previous simple chemistry DNS studies (Chakraborty et al., 2011; Chakraborty and Cant, 2013; Sellman et al., 2017), which suggested $\beta_{0}=8.0$ and $c_{c p}=0.55\left[1-\exp \left(-L e^{-2.5}\right)\right]$. It has been found for this database that the optimum values of these model parameters depend on the definition of RPV and they also change from one case to another. The optimum values of $\beta_{0}$ and $c_{c p}$ for cases A-C are listed in Table 2.

Figure 5 shows that eq. 5 accurately predicts $\left(T_{3}+T_{4}\right)$ for $\beta_{0}$ and $c_{c p}$ values listed in Table 2 in cases A-C. Previous analyses (Chakraborty et al., 2011; Chakraborty and Cant, 2013) indicated that $\beta_{0}$ and $c_{c p}$ depend on $L e$ and may also have some $R e_{t}$ dependence. This explains the different model parameters for different cases and for the choices of $\mathrm{H}_{2}, \mathrm{O}_{2}$ and $\mathrm{H}_{2} \mathrm{O}$, which have different diffusivities. It has also been shown (Chakraborty and Cant, 2005; Chakraborty et al., 2008; Chakraborty and Klein, 2008b) that the stretch rate dependence of $S_{d}$ 
significantly affects the statistical behaviours of the curvature and propagation terms, and Le influences these statistics as well. Although a single-step chemical mechanism captures the generic qualitative nature of the stretch rate dependence of $S_{d}$, these statistics are quantitatively different for different choices of RPV in $\mathrm{H}_{2}$-air flames (not shown here). Moreover, in $\mathrm{H}_{2}$-air flames $\dot{w}$ assumes its peak value at a much smaller value of $c$ for temperature-based RPV (for which $L e$ of the RPV is exactly equal to unity) than the value of $c$ at which $\dot{w}$ assumes its peak value for $\mathrm{H}_{2}, \mathrm{O}_{2}$ and $\mathrm{H}_{2} \mathrm{O}$ mass fraction-based RPVs (and also for single step irreversible Arrhenius type chemistry which is not shown here). Thus, the optimum values of $\beta_{0}$ and $c_{c p}$ show dependence on the choice of RPV, and these values are different from those reported previously (Hun and Huh, 2008; Chakraborty et al., 2011; Chakraborty and Cant, 2013) based on simple chemistry DNS analyses.

A comparison between the magnitudes of $T_{3}$ and $T_{4}$ in Fig. 1 reveals that the magnitude of $T_{4}$ is much greater than $T_{3}$ and thus eq. 5 principally models the unresolved part of the curvature term $T_{4}$. The curvature term $T_{4}$ can be split in terms of displacement speed components as (Chakraborty and Cant, 2007): $T_{4}=2 \overline{\left[\left(S_{r}+S_{n}\right) \kappa_{m}\right]} \Sigma_{s} \Sigma_{g e n}-4 \overline{\left(D \kappa_{m}^{2}\right)} \Sigma_{s} \Sigma_{g e n}$ where $S_{r}=\dot{w} /$ $\rho|\nabla c|$ and $S_{n}=\vec{N} \cdot \nabla(\rho D \vec{N} \cdot \nabla c) / \rho|\nabla c|$ are the reaction and normal diffusion components of displacement speed. According to Peters (2000), the contribution of $-4 \overline{\left(D \kappa_{m}^{2}\right)} \Sigma_{s} \Sigma_{g e n}$ strengthens with increasing $\mathrm{Ka}$. The magnitudes of the first two terms on the right hand side of eq. 5 remain small in comparison to $\left(-\beta_{0} \alpha_{N}\left(\bar{c}-c_{c p}\right) S_{L} \Sigma_{g e n}^{2} / \bar{c}(1-\bar{c})\right)$ and this term is principally responsible for sink contribution of $\left(T_{3}+T_{4}\right)$ (not shown here). Thus, the optimum value of $\beta_{0}$ shows an increasing trend from case $\mathrm{A}$ to case $\mathrm{C}$ in order to account for the strengthening contribution of $-4 \overline{\left(D \kappa_{m}^{2}\right)}{ }_{s} \Sigma_{g e n}$ with increasing $K a$. 
The observed lack of universal model parameter $\beta_{0}$ behaviour is attributed to the lack of the involvement of displacement speed $S_{d}$ and its curvature $\nabla \cdot \vec{N} / 2$ dependence in the in the model for $\left(T_{3}+T_{4}\right)=-\nabla \cdot\left[\left(S_{d} \vec{N}\right)_{S} \Sigma_{g e n}\right]+\overline{2\left(S_{d} \nabla \cdot \vec{N}\right)}{ }_{S} \Sigma_{g e n}$ given by eq. 5. As the displacement speed statistics including its curvature dependence change with the definition of RPV and also with the regime of combustion, the optimum value of $\beta_{0}$ shown in Table 2 exhibits dependence on the RPV definition and combustion regime.

\section{Mean reaction rate closure by $\Sigma_{g e n}$}

Finally, the closure of the mean reaction rate $\overline{\dot{w}}$ is discussed. First, in the context of RANS the contribution of $\overline{\nabla \cdot(\rho D \nabla c)}$ in statistically planar flames remains small in comparison with the mean reaction rate $\overline{\dot{w}}$, and thus $\overline{\dot{w}}$ can be modelled as: $\overline{\dot{w}} \approx{\overline{\left(\rho S_{d}\right)}}_{s} \Sigma_{g e n}$. The surface-averaged value of the density-weighted displacement speed $\overline{\left(\rho S_{d}\right)_{s}}$ is often approximated as $\overline{\left(\rho S_{d}\right)_{s}} \approx$ $\rho_{o} S_{L}$ (Boger et al., 1998; Hawkes and Cant 2001a; Hernandez-Perez et al., 2011; Ma et al., 2014).

The mean reaction rate $\overline{\dot{w}}$ obtained from DNS data is compared to $\rho_{0} S_{L} \Sigma_{g e n}$ in Fig. 6 for cases A-C for different choices of RPV. Figure 6 shows that $\rho_{0} S_{L} \Sigma_{g e n}$ captures the qualitative behaviour of $\overline{\dot{W}}$ for all cases for different choices of RPV. However, the extent of quantitative agreement depends on the case and also on the choice of RPV. In cases A and B, $\rho_{0} S_{L} \Sigma_{g e n}$ accurately predicts $\overline{\dot{W}}$ for the temperature based RPV, whereas $\rho_{0} S_{L} \Sigma_{g e n}$ slightly underpredicts (overpredicts) $\bar{w}$ towards the unburned (burned) gas side of the flame brush for RPVs defined based on the mass fractions of $\mathrm{H}_{2} \mathrm{O}$ and $\mathrm{O}_{2}$. However, $\rho_{0} S_{L} \Sigma_{\text {gen }}$ for the $\mathrm{H}_{2}$ mass fraction based RPV underestimates $\overline{\dot{W}}$, and the disagreement between $\rho_{0} S_{L} \Sigma_{g e n}$ and $\overline{\dot{w}}$ is the strongest for the $\mathrm{H}_{2}$ based RPV out of all choices of RPVs. Note that Le for $\mathrm{H}_{2}$ is 
significantly smaller than unity and previous analysis based on simple chemistry DNS (Chakraborty et al., 2011; Sellman et al., 2017) revealed that $\rho_{0} S_{L} \Sigma_{g e n}$ significantly underpredicts $\overline{\dot{W}}$ for RPVs with $L e \ll 1$. In case C, $\rho_{0} S_{L} \Sigma_{g e n}$ slightly overpredicts (underpredicts) $\overline{\dot{w}}$ towards the unburned (burned) gas side of the flame brush for all choices of RPV. This underprediction is most prominent in the case of the $\mathrm{H}_{2}$ mass fraction based RPV.

Figure 6 also shows that the quantitive prediction by $\rho_{0} S_{L} \Sigma_{g e n}$ becomes less accurate for case C. The effects of flame stretching strengthen with increasing $K a$ and ${\overline{\left(\rho S_{d}\right)}}_{s}$ cannot be approximated by $\rho_{o} S_{L}$ for the flames in the BRZ regime (e.g. case C). Recent studies (Klein $e t$ al., 2016; Chakraborty and Cant, 2007; Sabelnikov et al., 2017) showed that the approximation $\overline{\left(\rho S_{d}\right)_{s}} \approx \rho_{o} S_{L}$ leads to incorrect behaviour even for the CF and TRZ regimes of combustion, and thus improved modelling of $\overline{\left(\rho S_{d}\right)}$ is needed to extend the FSD-based closures for high Karlovitz number combustion and to ensure the fidelity of the prediction of $\overline{\dot{w}}$. Furthermore, Fig. 6 indicates that the transport equation of a single FSD might not be sufficient for $\bar{w}$ closures of all the major species for a multi-species system.

\section{Final comments on model performances for different regimes}

The optimal combinations of the closure models for the unclosed terms of the FSD transport equation for different combustion regimes for different definitions of RPVs are summarised in Table 3. It can be seen from Table 3 that the model performances for the unclosed terms of the FSD transport equation are comparable for different definitions of RPVs for terms $T_{1}, T_{21}, T_{22}$ but the optimal model for $T_{22}$ in case $\mathrm{C}$ is different to cases $\mathrm{A}$ and $\mathrm{B}$. Moreover, the model performances for $\left(T_{3}+T_{4}\right)$ and $\overline{\dot{w}}$ depend on the choice of RPV and shows the strongest deviations for $\mathrm{H}_{2}$ based RPV. It is worth noting that $|\nabla c|$ distributions for cases A-C are mostly similar for $\mathrm{O}_{2}$ and $\mathrm{H}_{2} \mathrm{O}$ based RPVs, whereas smaller values of $|\nabla c|$ are more likely in 
case C than in cases A and B (Chakraborty et al., 2018). This behaviour has been explained based on the differences in strain rate and displacement speed statistics for different species by Chakraborty et al. (2018). Case C nominally represents the broken reaction zones regime where energetic turbulent eddies penetrate into the flame structure and also in the reaction zone. This effect is particularly important for the $\mathrm{H}_{2}$ mass fraction based RPV because the reaction zone thickness is relatively thicker than $\mathrm{O}_{2}$ and $\mathrm{H}_{2} \mathrm{O}$ based RPVs due to smaller Lewis number of $\mathrm{H}_{2}$ than $\mathrm{O}_{2}$ and $\mathrm{H}_{2} \mathrm{O}$ (e.g. $L e_{\mathrm{H}_{2}} \ll 1$, and $L e_{\mathrm{O}_{2}}$ and $L e_{\mathrm{H}_{2} \mathrm{O}}$ are close to unity). Most existing FSD based models have been proposed for simple chemistry mechanisms and unity Lewis number conditions (Boger et al., 1998; Cant and Bray, 1998; Charlette et al., 2002; Keppeler et al., 2014; Klein et al., 2016; Chakraborty and Klein., 2008a; Ma et al., 2013; Cant et al., 1990; Candel et al., 1990; Duclos et al., 1993; Hawkes and Cant 2001a; Chakraborty and Cant, 2007; Hernandez-Perez et al., 2011; Reddy and Abraham, 2012; Chakraborty and Cant, 2013; Ma et al., 2014). Thus, it is perhaps not surprising that the existing models in some cases do not show a good agreement with DNS data for the $\mathrm{H}_{2}$ based RPV because $L e_{\mathrm{H}_{2}} \ll 1$, whereas the model performances for $\mathrm{O}_{2}$ and $\mathrm{H}_{2} \mathrm{O}$ based RPVs remain comparable because the Lewis numbers of $\mathrm{O}_{2}$ and $\mathrm{H}_{2} \mathrm{O}$ are close to unity.

\section{CONCLUSIONS}

The implications of the choice of RPV on the performances of the well-established sub-models of the $\Sigma_{g e n}$ transport and the FSD based $\overline{\dot{w}}$ closure have been analysed in the context of RANS using a detailed chemistry DNS database of freely-propagating thermo-diffusively neutral $\mathrm{H}_{2}$-air flames spanning the CF, TRZ and BRZ regimes of combustion. For this analysis, the RPV has been defined based on normalised $\mathrm{H}_{2}, \mathrm{O}_{2}$ and $\mathrm{H}_{2} \mathrm{O}$ mass fractions and also using the non-dimensional temperature. It has been found that the performances of the closures for turbulent flux of FSD, and tangential strain rate term remain mostly unaffected by the choice 
of RPV. However, both CPB and CFM models for the unresolved tangential strain rate term have been found not to perform well for the flames representing the CF and TRZ regimes of premixed combustion but the model performances are found to be relatively better for the flame belonging to the BRZ regime. The performance of a well-established existing model for the combined propagation and curvature terms has been found to be significantly dependent on the

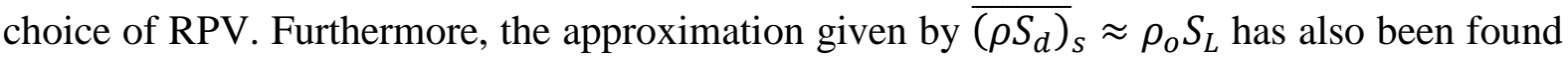
to be dependent on the choice of RPV and this approximation has been found to be especially unsatisfactory for all choices of RPV in the BRZ regime. Detailed explanations have been provided for the observed performances of the models for different combustion regimes and RPV definitions.

\section{ACKNOWLEDGEMENTS}

Part of the work presented in this study was sponsored by competitive research funding from King Abdullah University of Science and Technology (KAUST) and Engineering and Physical Sciences Research Council, UK The work made use of computational resources at KAUST Supercomputing Laboratory and ARCHER. 


\section{REFERENCES}

Arias, P.G., Chaudhuri, S., Uranakara, H.A., Im, H.G. 2016. Direct numerical simulations of statistically stationary turbulent premixed flame, Combust. Sci. Technol., 188, 1182.

Boger, M., Veynante, D., Boughanem, H., Trouvé, A. 1998. Direct Numerical Simulation analysis of flame surface density concept for Large Eddy Simulation of turbulent premixed combustion, Proc. Combust. Inst., 27, 917.

Burke, M.P., Chaos, M., Ju, Y. Dryer, F.L., Klippenstein, S.J. 2012. Comprehensive H2-O2 kinetic model for high-pressure combustion, Int. J. Chem. Kin., 44, 444.

Candel, S.M., Poinsot, T.J. 1990. Flame stretch and the balance equation for the flame area, Combust. Sci. Technol., 70, 1- 15.

Candel, S., Veynante, D., Lacas, F., Maistret, E., Darabhia, N., Poinsot, T. 1990. Coherent Flamelet Model: Applications and recent extensions, in: B.E. Larrouturou (Ed.), Recent Advances in Combustion Modelling, World Scientific, Singapore, 19.

Cant, R.S., Bray, K.N.C. 1988. Strained laminar flamelet calculations of premixed turbulent combustion in a closed vessel, Proc. Combust. Inst., 22, 791.

Cant, R.S., Pope, S.B., Bray, K.N.C. 1990. Modelling of flamelet surface to volume ratio in turbulent premixed combustion, Proc. Combust. Inst., 27, 809.

Chakraborty, N., Cant, R.S. 2005. Effects of strain rate and curvature on Surface Density Function transport in turbulent premixed flames in the thin reaction zones regime, Phys. Fluids, $17,65108$.

Chakraborty, N., Cant, R.S. 2006. Statistical behaviour and modelling of flame normal vector in turbulent premixed flames, Numer. Heat Trans. A, 50, 623.

Chakraborty, N., Cant, R. S. 2007. A priori analysis of the curvature and propagation terms of the flame surface density transport equation for large eddy simulation, Phys. Fluids, 19, 105101. 
Chakraborty, N., Cant, R.S. 2009a Physical insight and modelling for Lewis number effects on turbulent heat and mass transport in turbulent premixed flames, Numer. Heat Trans. A, $55,8,762$.

Chakraborty, N., Cant, R.S. 2009b Effects of Lewis number on turbulent scalar transport and its modelling in turbulent premixed flames, Combust. Flame, 156, 1427.

Chakraborty, N., Cant, R.S. 2011. Effects of Lewis number on Flame Surface Density transport in turbulent premixed combustion, Combust. Flame, 158, 1768.

Chakraborty, N., Cant, R.S. 2013. Turbulent Reynolds number dependence of Flame Surface Density transport in the context of Reynolds Averaged Navier Stokes Simulations, Proc. Combust. Inst., 34, 1347.

Chakraborty, N., Swaminathan, N. 2007. Influence of Damköhler number on turbulence-scalar interaction in premixed flames, Part I: Physical Insight, Phys. Fluids, 19, 045103.

Chakraborty, N., Klein, M. 2008a. A-priori direct numerical simulation assessment of algebraic flame surface density models for turbulent premixed flames in the context of large eddy simulation, Phys. Fluids, 20, 085108.

Chakraborty, N., Klein, M. 2008b. Influence of Lewis number on the Surface Density Function transport in the thin reaction zones regime for turbulent premixed flames, Phys. Fluids, 20, 065102.

Chakraborty, N., Hawkes, E.R., Chen, J.H., Cant, R.S. 2008. Effects of strain rate and curvature on Surface Density Function transport in turbulent premixed $\mathrm{CH}_{4}$-air and $\mathrm{H}_{2}$-air flames: A comparative study, Combust. Flame, 154, 259.

Chakraborty, N., Klein, M., Alwazzan, D., Im, H. G. 2018. Surface Density Function statistics in Hydrogen-air flames for different turbulent premixed combustion regimes, Combust. Sci. Technol., doi.org/10.1080/00102202.2018.1480015. 
Charlette, F., Meneveau, C., Veynante, D. 2002 A power law wrinkling model for LES of premixed turbulent combustion, Part I: Non dynamic formulation and initial tests, Combust. Flame, 131, 159.

Colin, O., Ducros, F., Veynante, D., Poinsot, T. 2000. A thickened flame model for large eddy simulations of turbulent premixed combustion, Phys. Fluids A, 12, 1843.

Colin, O, Benkenida A. and Angelberger, C., 2003. 3D Modeling of Mixing, Ignition and Combustion, Phenomena in Highly Stratified Gasoline Engines, Oil \& Gas Science and Technology - Rev. IFP, 58:1, 47-62.

Duclos, J.M., Veynante, D., Poinsot, T. 1993. A comparison of flamelet models for turbulent premixed combustion, Combust. Flame, 95, 101.

Hernandez-Perez, F.E., Yuen, F. T. C., Groth, C.P.T., Gülder, Ö. L. 2011. LES of a laboratoryscale turbulent premixed Bunsen flame using FSD, PCM-FPI and thickened flame models, Proc. Combust. Inst., 33, 1365.

Hawkes, E.R., Cant, R.S. 2001a. Implications of a flame surface density approach to large eddy simulation of premixed turbulent combustion, Combust. Flame, 126, 1617.

Hawkes, E.R., Cant, R.S. 2001b. Physical and numerical realizability requirements for flame surface density approaches to large-Eddy and reynolds averaged simulation of premixed turbulent combustion, Combust. Theory Model., 5, 699.

Hun, I., Huh, K.Y. 2008. Roles of displacement speed on evolution of flame surface density for different turbulent intensities and Lewis numbers in turbulent premixed combustion, Combust. Flame, 152, 194.

Im, H.G., Chen, J.H. 2002. Preferential diffusion effects on the burning rate of interacting turbulent premixed Hydrogen-Air flames, Combust. Flame, 126, 246. 
Katragadda, M., Malkeson, S.P., Chakraborty, N. 2011. Modelling of the tangential strain rate term of the Flame Surface Density transport equation in the context of Reynolds Averaged Navier Stokes Simulation, Proc. Combust. Inst., 33, 1429.

Keppeler, R., Tangermann, E., Allaudin, U., Pfitzner, M. 2014. LES of Low to High Turbulent Combustion in an Elevated Pressure Environment, Flow Turb. Combust., 92, 767.

Klein, M., Chakraborty, N., Pfitzner, M. 2016. Analysis of the combined modelling of subgrid transport and filtered flame propagation for premixed turbulent combustion, Flow Turb. Combust., 96, 921.

Klein, M., Kasten, C., Chakraborty, N., Im, H.G. 2018 Turbulent scalar fluxes in HydrogenAir premixed flames at low and high Karlovitz numbers, Combust. Theor. Modell., doi.org/10.1080/13647830.2018.1468034.

Knikker, R., Veynante, D., Meneveau, C. 2002. A priori testing of a similarity model for large eddy simulations of turbulent premixed combustion, Proc. Combust. Inst., 29, 2105.

Li S.C. and Kong Y.H., 2008. Diesel combustion modelling using LES turbulence model with detailed chemistry, Combust. Theory Model. 12:205-219.

Lindstedt, R.P., Vaos, E.M. 1999. Modelling of premixed turbulent flames with second moment methods, Combust. Flame, 116, 461.

Ma, T., Stein, T.O., Chakraborty, N., Kempf, A.M. 2013. A posteriori testing of algebraic flame surface density models for LES, Combust. Theor. Modell., 17, 431.

Ma, T., Stein, T.O., Chakraborty, N., Kempf, A.M. 2014. A-posteriori testing of the Flame Surface Density transport equation for LES, Combust. Theory Modell., 18, 32.

Meneveau, C., Poinsot, T. 1991. Stretching and quenching of flamelets in premixed turbulent combustion, Combust. Flame, 86, 311. 
Papapostolou, V., Chakraborty, N., Klein, M., Im, H. G. 2018.Statistics of scalar flux transport of major species in different premixed turbulent combustion regimes for turbulent $\mathrm{H} 2$-air flames, Proc. Turb. Heat and Mass Trans. 2018, 10th -13th July, Rio de Jeneiro, Brazil, 2018. Passot, T., Pouquet, A. 1987. Compressible Turbulence with a perfect gas law: A numerical. approach, J. Fluid Mech., 181, 441.

Peters, N. 2000. Turbulent Combustion, Cambridge Monograph on Mechanics, Cambridge University Press, Cambridge.

Reddy, H., Abraham, J. 2012. Two-dimensional direct numerical simulation evaluation of the flame-surface density model for flames developing from an ignition kernel in lean methane/air mixtures under engine conditions, Phys. Fluids, 24,105108.

Rogallo, R.S. 1981. Numerical experiments in homogeneous turbulence, NASA Technical Memorandum 81315, NASA Ames Research Center, California.

Sabelnikov, V., Lipatnikov, A.N., Chakraborty, N., Nishiki, S., Hasagawa, T. 2017. A balance equation for the mean rate of product creation in premixed turbulent flames, Proc. Combust. Inst., 36, 1893.

Sellmann, J., Lai, J., Chakraborty, N., Kempf, A.M. 2017. Flame Surface Density based modelling of head-on quenching of turbulent premixed flames, Proc. Combust. Inst., 36, 1817. Veynante, D., Piana, J., Duclos, J.M., Martel, C. 1996. Experimental analysis of flame surface density models for premixed turbulent combustion, Proc. Combust. Inst., 26, 413.

Veynante, D., Trouvé, A., Bray, K.N.C., Mantel, T. 1997. Gradient and counter-gradient scalar transport in turbulent premixed flames, Proc. Combust. Inst., 26, 413.

Vermorel O., Richard, S., Colin, O., Angelberger, C., Benkenida, A. and Veynante, D. 2009. Towards the understanding of cyclic variability in a spark ignited engine using multicycle LES, Combust. Flame 156:525-1541. 
Wacks, D.H., Chakraborty, N., Klein, M., Arias, P.G., Im, H.G. 2016. Flow topologies in Podifferent regimes of premixed turbulent combustion: A direct numerical simulation analysis, Phys. Rev. F, 1, 083401.

Yoo, C.S., Wang, Y., Trouve, A., Im, H.G. 2005. Characteristic boundary conditions for direct simulations of turbulent counterflow flames, Combust. Theor. Modell., 9, 617.

Yeung, P.K., Girimaji, S.S., Pope, S.B. 1990. Straining and scalar dissipation on material surfaces in turbulence: implications for flamelets, Combust. Flame, 79, 340. 


\section{TABLES}

\begin{tabular}{|c|c|c|c|c|c|}
\hline Case & $u^{\prime} / S_{L}$ & $l_{T} / \delta_{t h}$ & $R e_{t}$ & Da & Ka \\
\hline A & 0.7 & 14.0 & 227 & 20.0 & 0.75 \\
\hline B & 5 & 14.0 & 1623 & 2.8 & 14.4 \\
\hline C & 14 & 4.0 & 1298 & 0.29 & 126 \\
\hline
\end{tabular}

Table 1: List of inflow turbulence parameters

\begin{tabular}{|c|c|c|c|c|c|}
\hline & $\begin{array}{c}\text { Model } \\
\text { parameters }\end{array}$ & $\boldsymbol{c}\left(\mathbf{H}_{2}\right)$ & $\boldsymbol{c}\left(\mathbf{O}_{\mathbf{2}}\right)$ & $\boldsymbol{c}\left(\mathbf{H}_{\mathbf{2}} \mathbf{0}\right)$ & $\boldsymbol{c}(\mathbf{T})$ \\
\hline \multirow{2}{*}{ Case A } & $\beta_{0}$ & 4.0 & 4.5 & 2.5 & 4.0 \\
\cline { 2 - 6 } & $c_{c p}$ & 0.45 & 0.65 & 0.3 & 0.4 \\
\hline \multirow{2}{*}{ Case B } & $\beta_{0}$ & 3.5 & 4.0 & 3.0 & 4.0 \\
\cline { 2 - 6 } & $c_{c p}$ & 0.2 & 0.6 & 0.3 & 0.2 \\
\hline Case C & $\beta_{0}$ & 11.0 & 4.0 & 8.0 & 11.0 \\
\cline { 2 - 6 } & $c_{c p}$ & 0.1 & 0.1 & 0.2 & 0.1 \\
\hline
\end{tabular}

Table 2: Optimum values of $\beta_{0}$ and $c_{c p}$ for different RPV definitions for cases A-C 


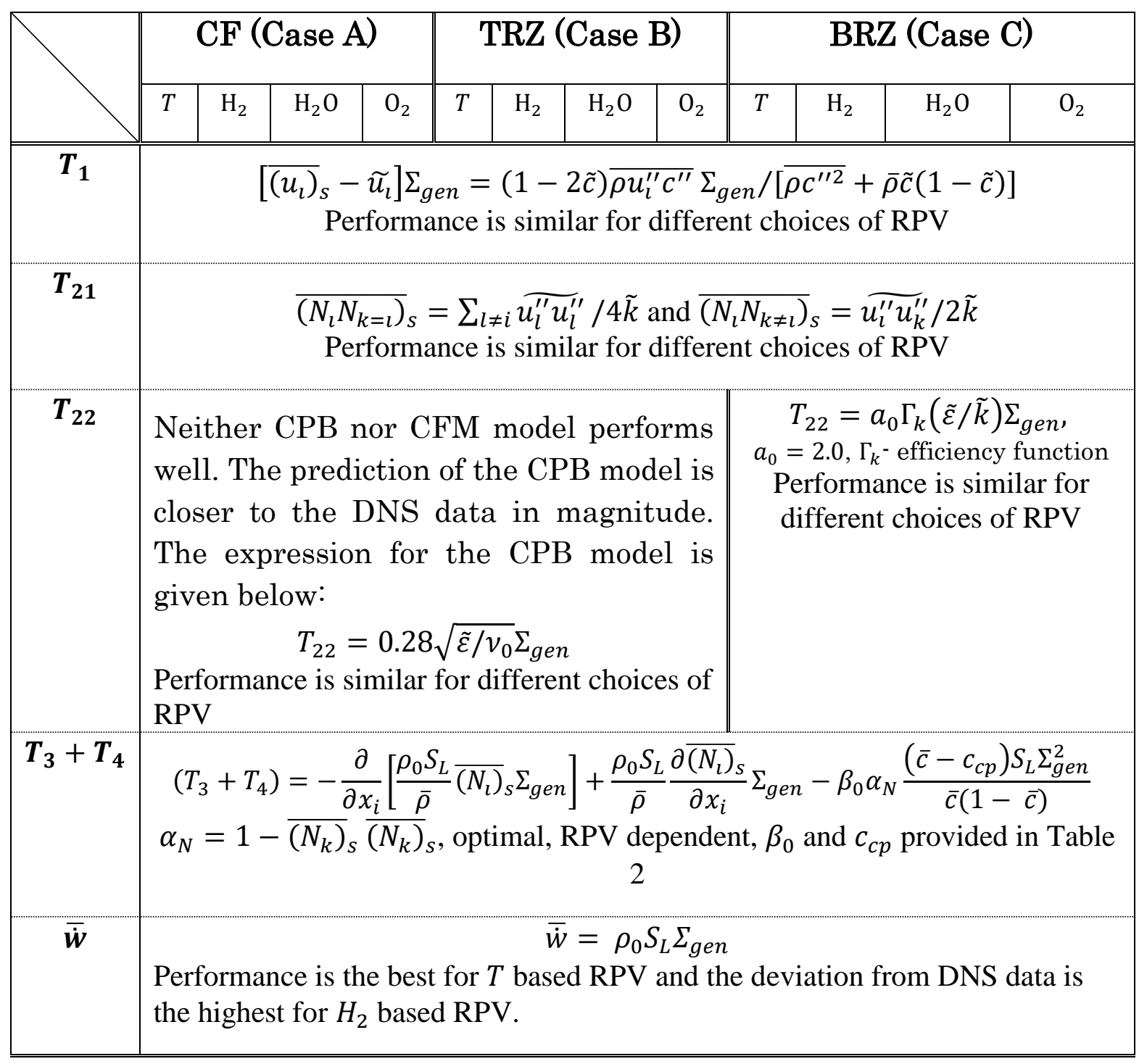

Table 3: Summary of optimal combination of the closure models for the unclosed terms of the FSD transport equation for different combustion regimes for different definitions of RPVs. 


\section{FIGURE CAPTIONS}

Fig. 1: Variations of $T_{i} \times \delta_{t h}^{2} / S_{L}$ (where solid line, dashed line, dashed dotted line, and line with circles, are used for $i=1-4$ respectively) with $\tilde{c}$ for cases A-C from left to right columns respectively. The results in Figs. 1-6 for RPV definitions based on temperature, $H_{2}$, $\mathrm{H}_{2} \mathrm{O}$ and $\mathrm{O}_{2}$ mass fractions are shown by black, red, green and blue lines respectively.

Fig. 2: Variation of $\left[\overline{\left(u_{l}\right)_{S}}-\tilde{u}_{i}\right] \Sigma_{\text {gen }} \times \delta_{t h} / S_{L}$ (solid lines for DNS data) along with the predictions of the gradient hypothesis (dotted line) and eq. 3 (broken line) with $\tilde{c}$ for cases AC from left to right columns respectively.

Fig. 3: Variation of $T_{21} \times \delta_{t h}^{2} / S_{L}$ (solid lines for DNS data) along with the predictions of the CPB (broken line) and VPDM (dotted line) models with $\tilde{c}$ for cases A-C from left to right columns respectively.

Fig. 4: Variation of $T_{22} \times \delta_{t h}^{2} / S_{L}$ (solid lines for DNS data) along with the predictions of the CPB (broken line) and CFM (dotted line) models with $\tilde{c}$ for cases A-C from left to right columns respectively.

Fig. 5: Variation of $\left(T_{3}+T_{4}\right) \times \delta_{t h}^{2} / S_{L}$ (solid lines for DNS data) along with the predictions of eq. 5 with $\beta_{0}$ given in Table 2 (broken line) with $\tilde{c}$ for cases $\mathrm{A}-\mathrm{C}$ from left to right columns respectively.

Fig. 6: Variations of $\overline{\dot{w}} \times \delta_{t h} / \rho_{0} S_{L}$ (solid line), along with $\rho_{0} S_{L} \Sigma_{g e n} \times \delta_{t h} / \rho_{0} S_{L}$ (broken line) with $\tilde{c}$ for cases A-C from left to right columns respectively. 

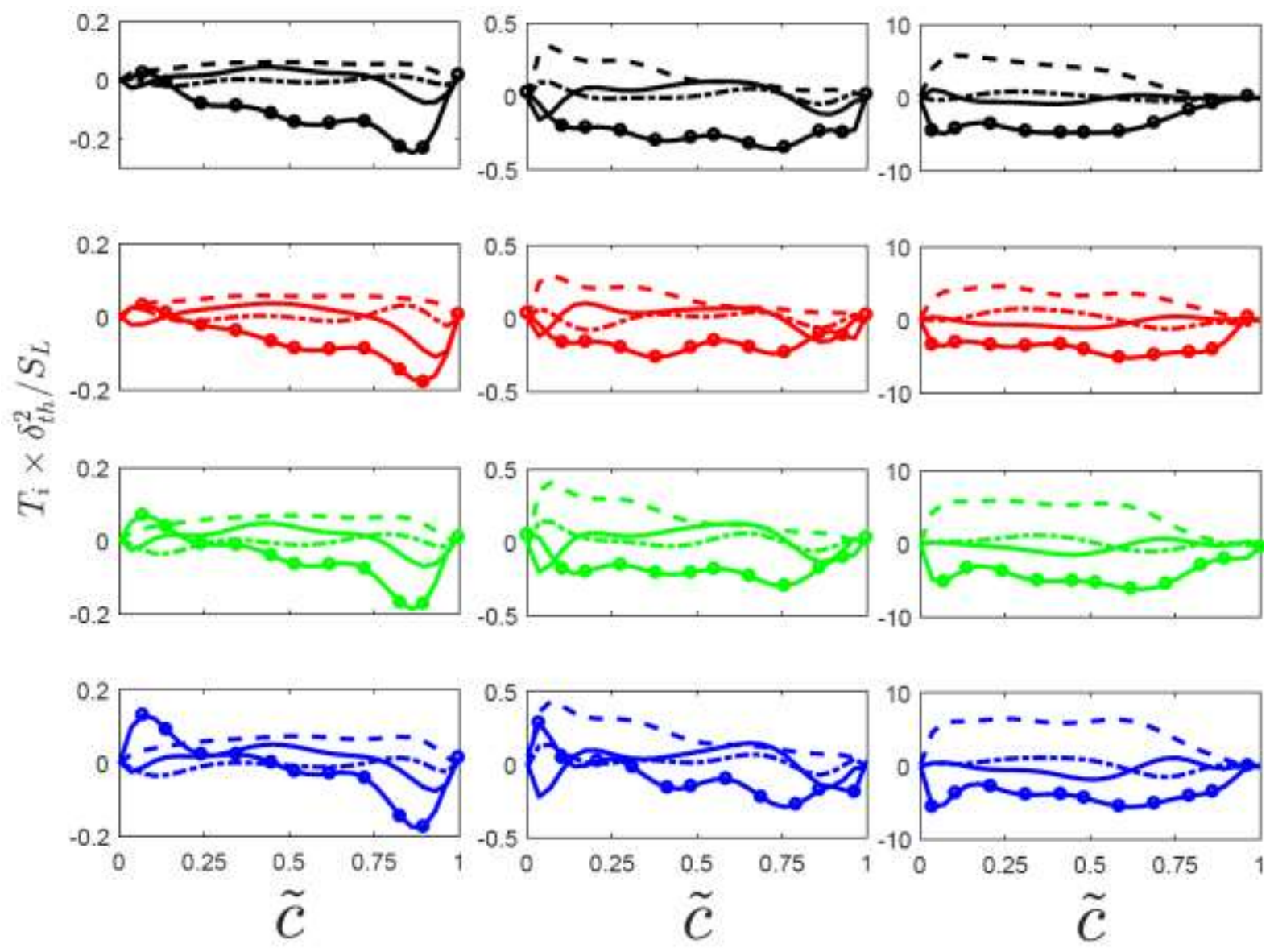

Fig. 1: Variations of $T_{i} \times \delta_{t h}^{2} / S_{L}$ (where solid line, dashed line, dashed dotted line, and line with circles, are used for $i=1-4$ respectively) with $\tilde{c}$ for cases A-C from left to right columns respectively. The results in Figs. 1-6 for RPV definitions based on temperature, $H_{2}$, $\mathrm{H}_{2} \mathrm{O}$ and $\mathrm{O}_{2}$ mass fractions are shown by black, red, green and blue lines respectively. 

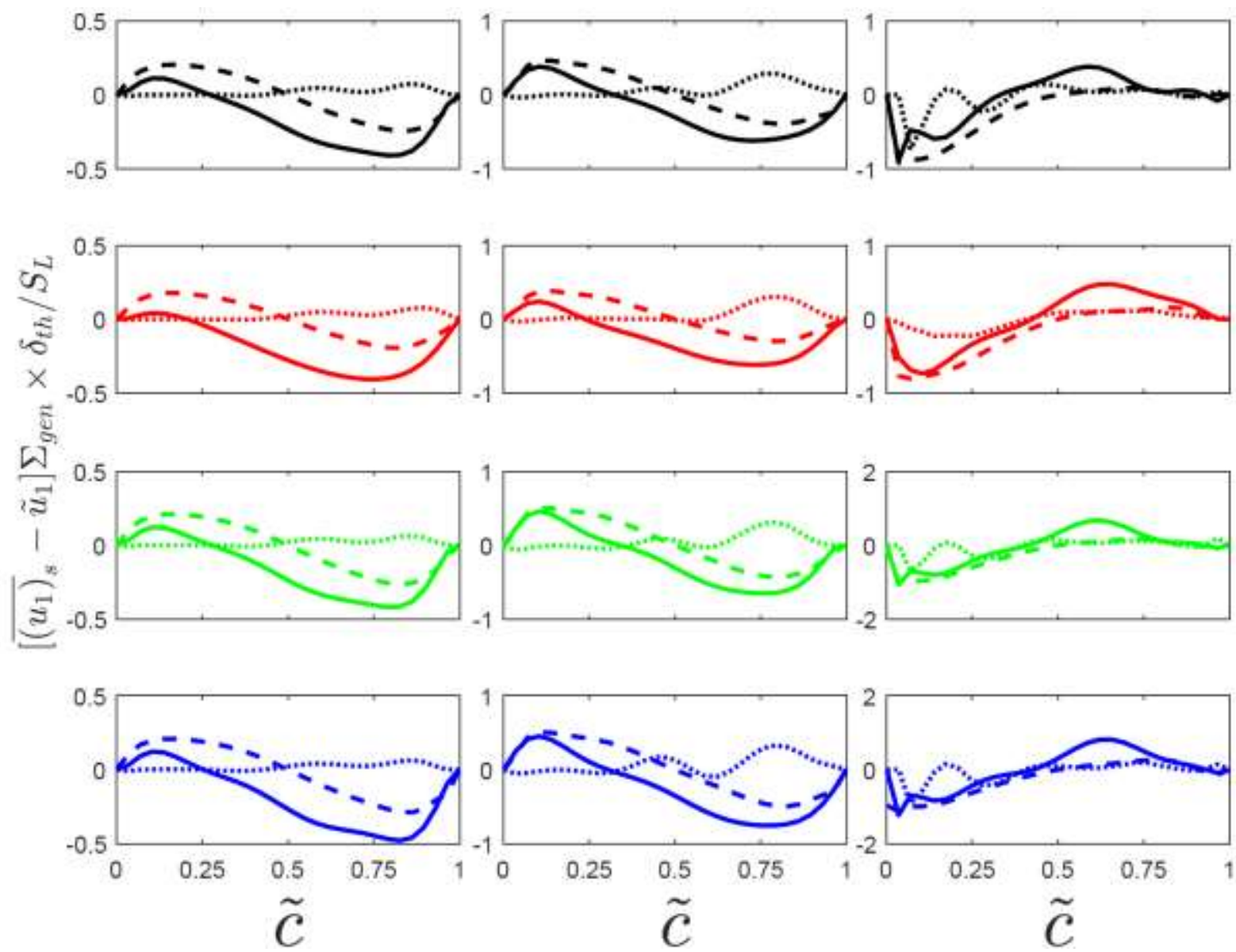

Fig. 2: Variation of $\left[\left(u_{l}\right)_{S}-\tilde{u}_{i}\right] \Sigma_{\text {gen }} \times \delta_{t h} / S_{L}$ (solid lines for DNS data) along with the predictions of the gradient hypothesis (dotted line) and eq. 3 (broken line) with $\tilde{c}$ for cases A$\mathrm{C}$ from left to right columns respectively. 

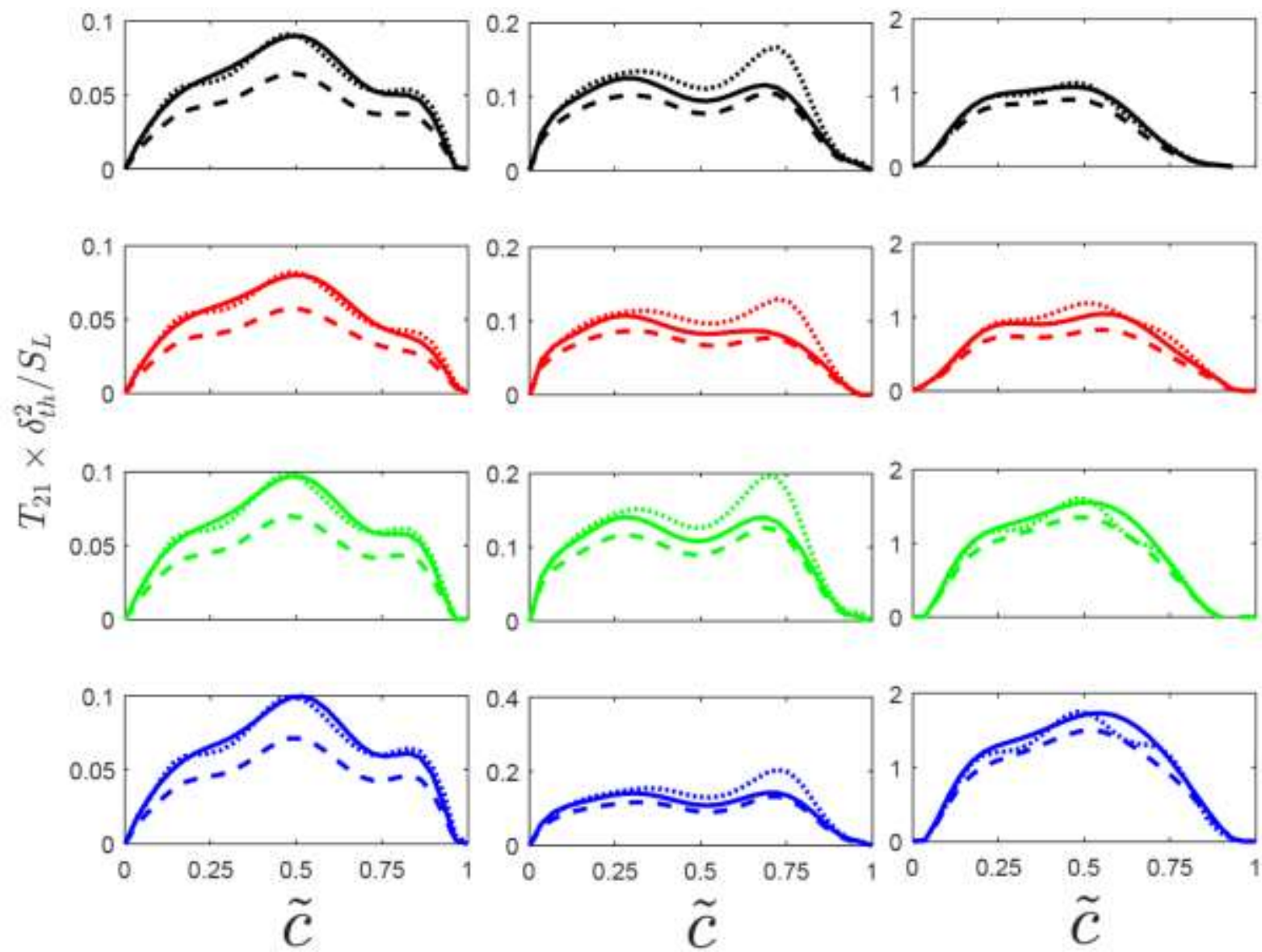

Fig. 3: Variation of $T_{21} \times \delta_{t h}^{2} / S_{L}$ (solid lines for DNS data) along with the predictions of the $\mathrm{CPB}$ (broken line) and VPDM (dotted line) models with $\tilde{c}$ for cases A-C from left to right columns respectively. 

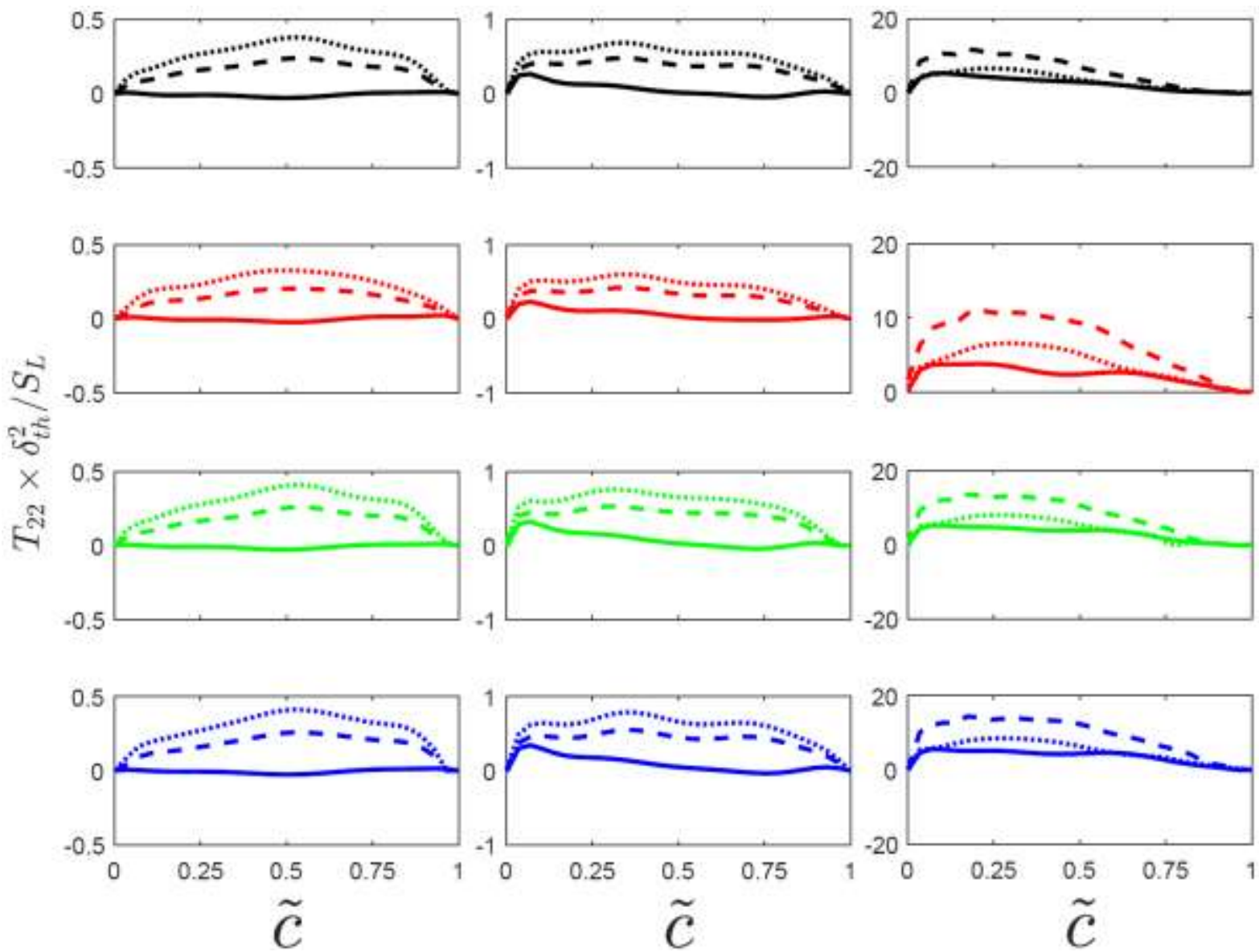

Fig. 4: Variation of $T_{22} \times \delta_{t h}^{2} / S_{L}$ (solid lines for DNS data) along with the predictions of the CPB (broken line) and CFM (dotted line) models with $\tilde{c}$ for cases A-C from left to right columns respectively. 

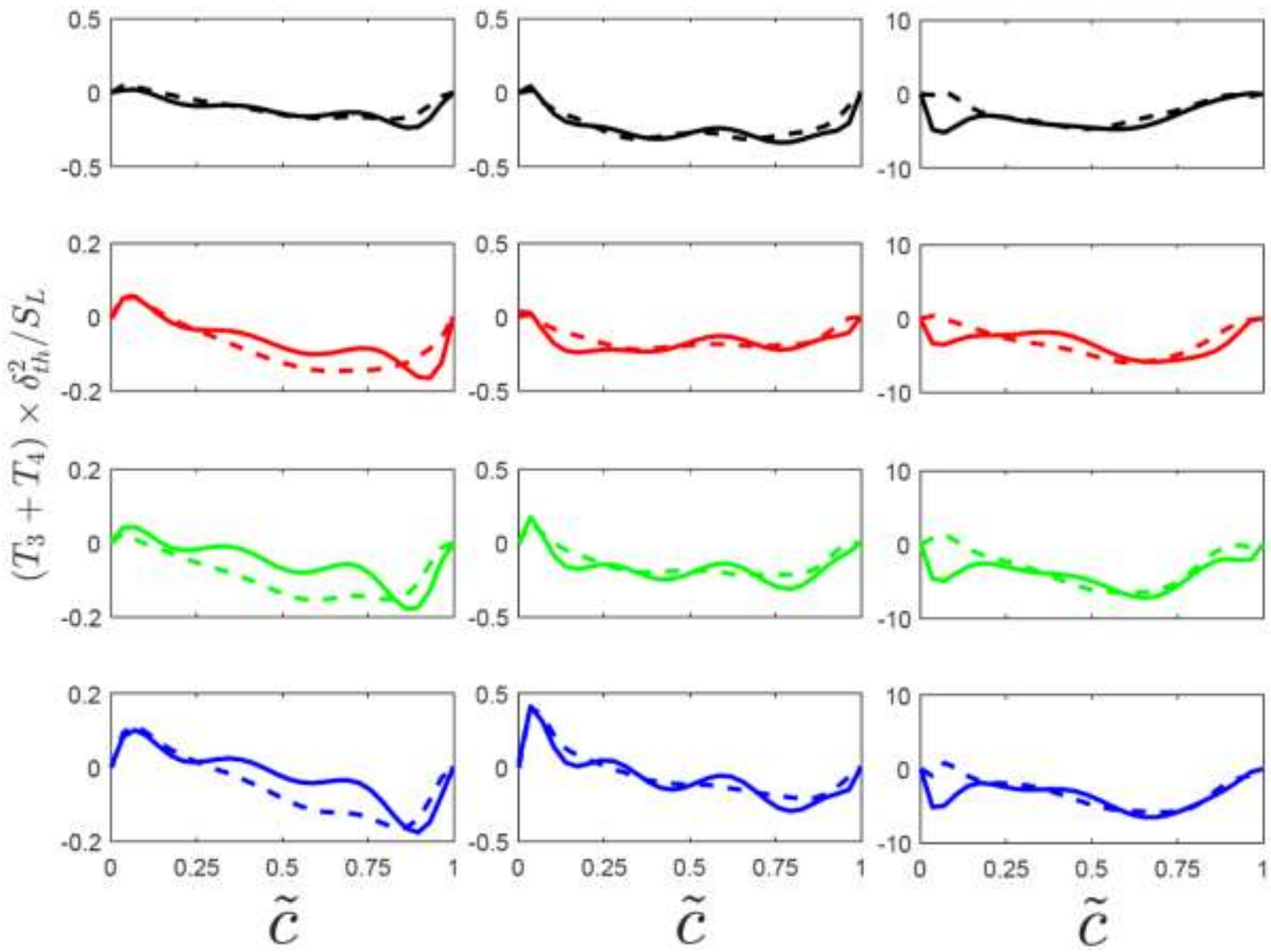

Fig. 5: Variation of $\left(T_{3}+T_{4}\right) \times \delta_{t h}^{2} / S_{L}$ (solid lines for DNS data) along with the predictions of eq. 5 with $\beta_{0}$ given in Table 2 (broken line) with $\tilde{c}$ for cases $\mathrm{A}-\mathrm{C}$ from left to right columns respectively. 

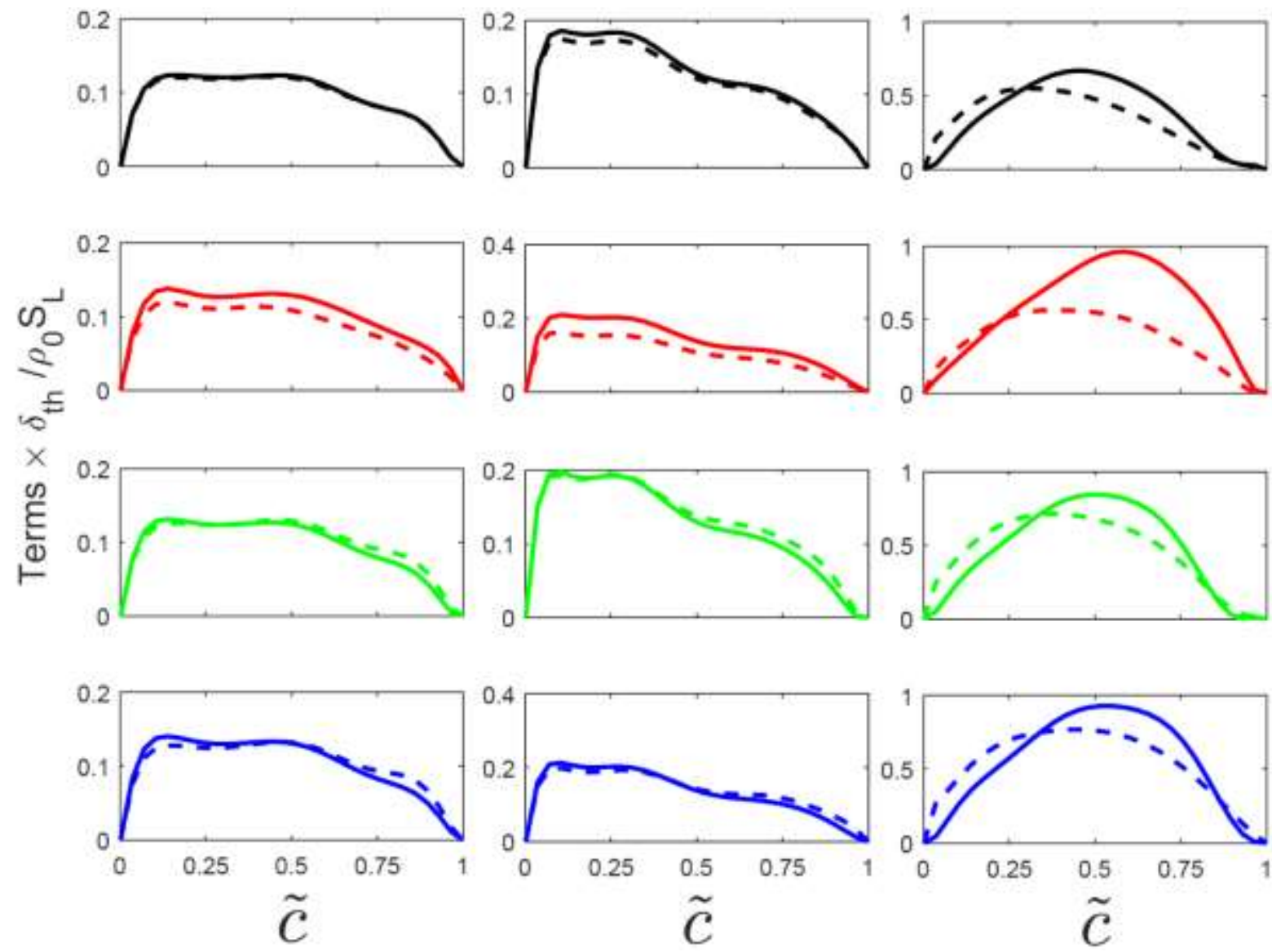

Fig. 6: Variations of $\overline{\dot{w}} \times \delta_{t h} / \rho_{0} S_{L}$ (solid line), along with $\rho_{0} S_{L} \Sigma_{g e n} \times \delta_{t h} / \rho_{0} S_{L}$ (broken line) with $\tilde{c}$ for cases A-C from left to right columns respectively. 